\title{
Artificial Intelligence Applications of Hazardous Mitigation for Transport Aircraft in Severe Atmospheric Turbulence
}

\author{
Ray C. Chang* \\ Department of Aviation Mechanical Engine, China University of Science and Technology, Taiwan \\ ${ }^{*}$ Corresponding author
}

\begin{abstract}
This paper presents a new study of artificial intelligence applications through the simulation of improved control strategy to provide the mitigation concepts and formulate preventive actions for aircraft operation. The fuzzy-logic modeling (FLM) technique is used to establish flight control models with the function of nonlinear dynamic inversion based on the datasets from the flight data recorder (FDR). The improved control strategy can be obtained through the simulations of flight control models to enhance the stability and controllability before and during the severe atmospheric turbulence.
\end{abstract}

Keywords-atmospheric turbulence; plunging motion; flight data recorder; nonlinear dynamic inversion

\section{INTRODUCTION}

The transport aircraft in flight is subjected to atmospheric turbulence resulting in rapidly varying aerodynamic and flight dynamic characteristics. These varying characteristics not only pose threats to flight safety, but also may cause structural damages and reduce fatigue life [1]. These effects of varying characteristics are quite difficult to be simulated by windtunnel tests or conventional flight simulators.

The evaluations of stability characteristics during sudden plunging motion through oscillatory derivatives extracted from fuzzy-logic aerodynamic models along the flight path for a transport aircraft encountering atmospheric turbulence were demonstrated in references of [2, 3]. The use of oscillatory derivative instead of steady damping ones was more consistent with the actual case of the aerodynamic damping in the analysis of stability characteristics in these references.

To examine possible mitigation concepts of accident prevention, a new study of artificial intelligence applications through the simulations of improved control strategy before and during the severe atmospheric turbulence encounters is undertaken. This paper employs fuzzy-logic modeling technique to establish flight control models with the function of nonlinear dynamic inversion based on the datasets from the flight data recorder (FDR). The improved control strategy can be obtained through the flight control models. Then, the simulated results of hazardous mitigation can be determined based on the influence of crosswind, caused reasons of significant increase in angles of attack, and flight operations. The necessary and appropriate applications to improve the flight controls of rudder, aileron, and elevator before and during severe atmospheric turbulence are demonstrated.

\section{NUMERICAL METHOD DEVELOPMENT}

\section{A Fuzzy-Logic Technique}

Modeling procedures start from setting up numerical relations between the input (i.e. flight variables) and output (i.e. flight operations or aircraft response). To obtain continuous variations of predicted results, the present method is based on the internal functions, instead of fuzzy sets [4], to generate the output of the model.

System identification in the present paper includes two tasks: one is the model structure identification, and another one is to identify the parameters that represent their corresponding model structures. The present modeling method was first developed by Takagi \& Sugeno in 1985, and later in 1995, Tan \& Xie [5] applied the theory to simulate microelectronic processes with very good accuracy. The present paper is based on the modeling technique suggested by Tan \& Xie [6]. This technique was first applied to aviation technology in 1997 and later to flight data [7]. A detailed description of the fuzzy-logic algorithm is available in reference [8].

\section{B Flight Control Model}

A preprocessor is needed to re-arrange the flight variables in the dataset, and interpolate the variables if necessary by a monotone cubic spline [9]. For example, the FDR data can provide the necessary data to develop models for pilot training and flight control development. If flight test data are used, no further interpolation is necessary in most cases. To illustrate, the roll oscillation and sudden plunging motion problems will be considered. The following numerical models based on the FDR data in cruise flight operation with atmospheric turbulence encountering may be generated:

$$
\begin{gathered}
\delta_{a}=f_{1}\left(\alpha, \theta, \phi, \dot{\phi}, \dot{\psi}, \dot{\theta}, V_{c a s}, a_{z}, a_{y}, V_{c r w}, V_{t l w}, R \text { Mic }\right) \\
\delta_{r}=f_{1}\left(\alpha, \theta, \phi, \dot{\phi}, \dot{\psi}, \dot{\theta}, V_{c a s}, a_{z}, a_{y}, V_{c r w}, V_{t l w}, \text { YMic }\right)
\end{gathered}
$$




$$
\delta_{e}=f_{1}\left(\alpha, \theta, \phi, \dot{\phi}, \dot{\psi}, \dot{\theta}, V_{c a s}, a_{z}, a_{y}, V_{c r w}, V_{t l w}, \text { PMic }\right)
$$

where the parameter on left hand side of Eqs. (1) (3) represents the aileron angle $(\delta a)$, rudder angle $(\delta r)$, and elevator angles $\left(\delta_{e}\right)$. The flight variables on the right hand side represent angle of attack $(\alpha)$, pitch angle $(\theta)$, roll angle $(\phi)$, time rate of roll angle ( $\stackrel{\&}{\phi})$, time rate of heading angle $(\dot{\psi})$, time rate of pitch angle $(\dot{\theta})$, calibrated airspeed $\left(V_{\text {cas }}\right)$, vertical accelerations (az), lateral acceleration (ay), crosswind speed (Vcrw), tailwind speed (Vtlw), rolling moment due to inertial coupling (RMic), yawing moment due to inertial coupling (YMic), and pitching moment due to inertial coupling (PMic).

In the sudden plunging motion, $\dot{\theta}$ is the enabling variable because it is the response directly related to elevator. However, associated with the specified change in $\alpha$, the lateral acceleration (ay) and vertical accelerations (az) should be changed as well. Therefore, the following sub-models are needed associated with Eq. (3):

$$
\begin{gathered}
\alpha=f_{11}\left(\theta, \phi, \stackrel{\&}{\phi} \stackrel{\&}{\&} \stackrel{\&}{\&}, V_{c a s}, a_{y}, V_{c r w}, V_{t l w}, \text { PMic }\right) \\
a_{y}=f_{12}\left(\alpha, \theta, \phi, \stackrel{\&}{\phi} \stackrel{\&}{\psi} \stackrel{\&}{\theta}, V_{c a s}, a_{z}, V_{c r w}, V_{t l w}, \text { PMic }\right) \\
a_{z}=f_{13}\left(\alpha, \theta, \phi, \stackrel{\&}{\phi} \stackrel{\&}{\psi} \stackrel{\&}{\theta}, V_{c a s}, a_{y}, V_{c r w}, V_{t l w}, \text { PMic }\right)
\end{gathered}
$$

\section{NUMERICAL RESULTS AND DISCUSSIONS}

\section{A. Flight Data}

In the present paper, a twin-jet transport is employed; this transport encountered atmospheric turbulence during the cruise flight at altitude about $10,050 \mathrm{~m}$. The dataset used for reconstructions are extracted from the FDR during turbulence encounter lasting for 92 seconds.

The main aircraft geometric and inertial characteristics for this transport are taken to be:

$$
\begin{aligned}
& W \text { (take-off })=1,431,800 \mathrm{~N}(321900 \mathrm{lb}) \\
& S=260 \mathrm{~m}^{2}\left(2798.7 \mathrm{ft}^{2}\right), \bar{C}=6.608 \mathrm{~m}(21.68 \mathrm{ft}), b=44.827 \\
& \mathrm{~m}(147.08 \mathrm{ft}) \\
& I_{x x}=10,710,000 \mathrm{~kg}-\mathrm{m}^{2}\left(7,899,900 \text { slugs- } \mathrm{ft}^{2}\right), I_{y y}= \\
& 14,883,800 \mathrm{~kg}-\mathrm{m}^{2}\left(10,978,000 \text { slugs- } \mathrm{ft}^{2}\right) \\
& I_{z z}=25,283,271 \mathrm{~kg}-\mathrm{m}^{2}\left(18,648,470 \text { slugs- } \mathrm{ft}^{2}\right), I_{x z}=0.0 \mathrm{~kg}-
\end{aligned}
$$$$
\mathrm{m}^{2}
$$

The necessary data in the FDR to reconstruct the aerodynamic and flight environments for this transport are time $(\mathrm{t})$, CAS, pressure altitude (h) or radio altitude, Euler angles ( $\phi$, $\theta$, and $\psi$ ), the longitudinal, lateral, and vertical accelerations (ax, ay, az), angle of attack $(\alpha)$, outside air temperature, wind speed, wind direction, ground speed, and drift angle. Since only the normal acceleration is recorded in $8-\mathrm{Hz}$ resolution (i.e. 8 points per second), all other parameters are interpolated with a monotone cubic spline to the same sampling rate.

\section{B. Flight Environments}

The transport encountered severe atmospheric turbulence in revenue flight. As a result, several passengers and cabin crews sustained injuries, because of which this event was classified as the aviation accident. To examine the flight dynamic mechanism of plunging motion, it is imperative to understand the flight environments in detail.

The corresponding flight data of this transport with severe atmospheric turbulence encountered at the altitude around $10,050 \mathrm{~m}$ in transonic flight is presented in Figure. 1. The variation of vertical acceleration is presented in Figure. 1(a), showing the highest az being $1.75 \mathrm{~g}$ around $\mathrm{t}=3935 \mathrm{sec}$ and the lowest being $0.02 \mathrm{~g}$ around $\mathrm{t}=3937 \mathrm{sec}$. Figure. 1(b) shows that variation of $\alpha$ is approximately in phase with az. When az is the highest (around $t=3935$ sec.), the autopilot was deactivated (A/P tripped) at $\mathrm{t}=3935 \sim 3944$ sec., for 9 seconds; the aircraft rapidly plunging downward with the drop-off height of $67.1 \mathrm{~m}$ as shown in Figure I (c); and $\alpha$ is highest about 7.1 deg. in Figure I (b). At the same time, $\mathrm{M}$ is around 0.77 in Figure I (d).
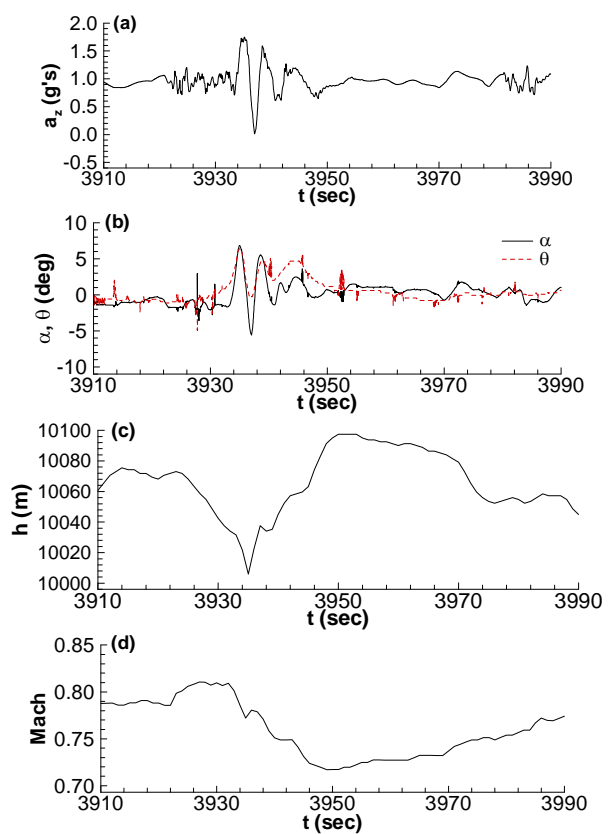

FIGURE I. TIME HISTORY OF FLIGHT VARIABLES FOR THE TRANSPORT IN SEVERE ATMOSPHERIC TURBULENCE

\section{Mitigation of Crosswind Effects}

Before the severe atmospheric turbulence encountered, the transport is subjected crosswind with considerable magnitude, so that operational actions of rudder ( $\delta \mathrm{r}$ ) after $\mathrm{t}>3930 \mathrm{sec}$. is constantly applied to counter the motion caused by the crosswind, as shown in Figure II. The variations of time rates 
of heading angles $(\stackrel{\&}{\Psi})$ and lateral acceleration (ay) are very large in the period of $t=3933 \sim 3939$ sec. as shown by the solid lines in Figure III and Figure IV, respectively. The large variations of time rates of heading angles $(\stackrel{\&}{\Psi})$ and lateral acceleration (ay) in the period of $\mathrm{t}=3933 \sim 3939$ sec are reduced, as shown by dotted lines in Figure II and Figure III, respectively, after the improvements of rudder operations. There are significant differences between the original rudder and improved rudder control in Figure II; the dotted line represents improved rudder control.

The original roll angle ( $\phi)$ is represented by the solid line in Figure V. The value of $\phi$ changes from positive to negative at $\mathrm{t}=3834$ sec.; the values are negative in the period of $\mathrm{t}=3934$ $\sim 3940$ sec.; when the crosswind suddenly increases, the value of $\phi$ reaches -19 deg.; the variations of $\phi$ are changed rapidly in the time period of $\mathrm{t}=3934 \sim 3945$ sec. The sensitivity analysis of $\phi_{\text {variations are presented in Figure. 6; the original }}$ time rate of roll angle $(\phi)$ is represented by the solid line; the maximum value is close to $25 \mathrm{deg} / \mathrm{sec}$ at $\mathrm{t}=3940 \mathrm{sec}$. The response of aileron control against $\phi$ variations is shown in Figure VII; the original time rate of roll angle is represented by the solid line; the highest value is around 12 deg. at $t=3940$ sec. The roll angle variations due to the crosswind effects are shown in Figure V and aileron control in Figure VII, therefore the varying crosswind to induce rolling motion can be made as the conclusion in this section. The large variations of original roll angle and original time rates of roll angle in the period of $t=$ $3936 \sim 3945$ sec are reduced, as shown by dotted lines in Figure $\mathrm{V}$ and Figure VI, respectively, after the improvements of aileron control. There are significant differences between the original and improved aileron control in Figure II; the dotted line represents improved aileron control.

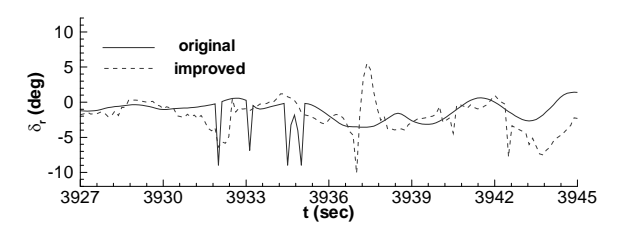

FIGURE II. ORIGINAL AND IMPROVED RUDDER CONTROL

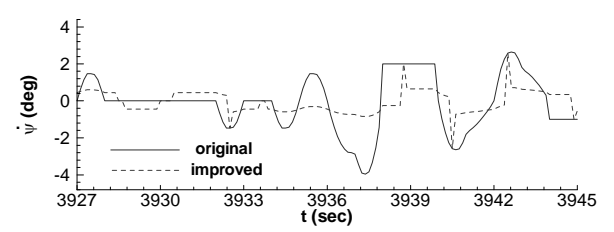

FIGURE III. ORIGINAL AND IMPROVED TIME RATES OF HEADING ANGLES

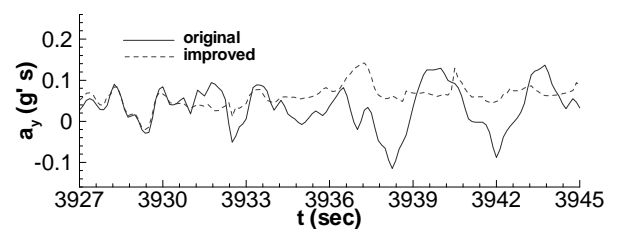

Figure IV. ORIGINAL AND IMPROVED LATERAL ACCELERATION

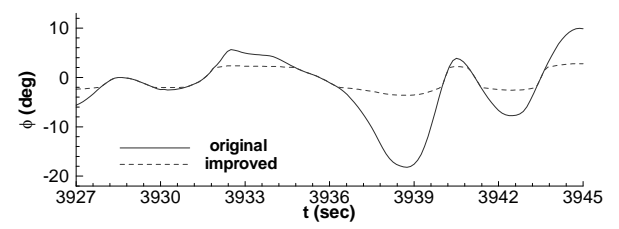

FIGURE V. ORIGINAL AND IMPROVED ROLL ANGLE

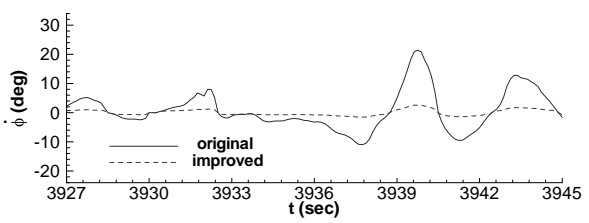

FIGURE VI. ORIGINAL AND IMPROVED TIME RATE OF ROLL ANGLE

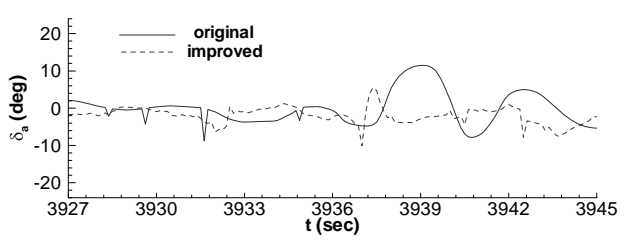

FIGURE VII ORIGINAL AND IMPROVED AILERON CONTROL

\section{Mitigation of Significant Increase in Angles of Attack}

Figure VIII Figure XII present the original and improved angles of attack, pitch angles, time rate of pitch angles, vertical accelerations, and elevator angles; the solid lines represent the original flight data. The largest angle of attack reached is 7.1 deg around $t=3935$ sec and the time history of angle of attack is increased at first and reduced afterward as shown in Figure. 8. The time history of pitch angles, time rate of pitch angle, and vertical accelerations are also increased first and then decreased to correspond with the angle of attack as shown in Figure VIIII, Figure X, and Figure XI, respectively. The solid lines in Figure 12 , the elevator angles before $t=3930 \mathrm{sec}$ were basically no elevator activity; in the period of $\mathrm{t}=3930 \sim 3945 \mathrm{sec}$, almost all the values of elevator are positive, except the values of elevators are negative in the period of $\mathrm{t}=3935 \sim 3937$ sec. Positive values of elevator represent nose-down elevator activities; the magnitudes of angles of attack and pitch angles should be decreased or negative.

The large magnitudes of angles of attack, pitch angles, time rate of pitch angles, and vertical accelerations in the period of $t$ $=3933 \sim 3945$ sec are reduced, as shown by dotted lines in Figure VIII Figure XI, respectively, after the improvements of elevator control. The dotted line magnitudes of elevator angles are reduced after $\mathrm{t}=3936 \mathrm{sec}$ in Figure XII; it represents 
smooth operation of elevator angles during the plunging motion.

The conditions of sudden increase and abrupt decrease in angles of attack are mitigated; the largest angle of attack 7.1 deg. is reduced to 2.3 deg. around $t=3935 \mathrm{sec}$ as shown by the dotted lines in Figure VIII. The large magnitudes of pitch angles and time rate of pitch angles are also mitigated as shown by the dotted lines in Figure IX and Figure X, respectively. The transport aircraft rapidly plunging downward with vertical accelerations are reduced from 1.76 to 1.22 in the period of $\mathrm{t}=$ 3933 3936 sec and plunging upward with vertical accelerations are increases from 0.17 to 0.61 in the period of $\mathrm{t}=$ 3936 3939 sec as shown by the dotted lines in Figure XI.

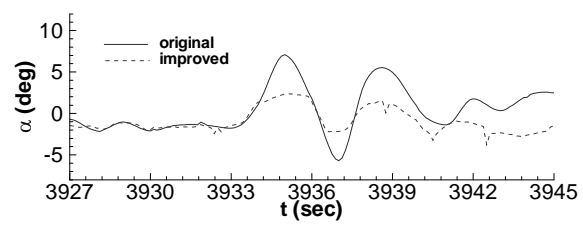

FIGURE VIII. ORIGINAL AND IMPROVED ANGLE OF ATTACK

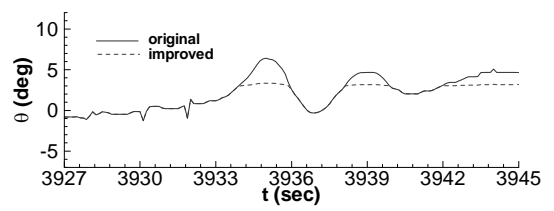

FIGURE IX. ORIGINAL AND IMPROVED PITCH ANGLE

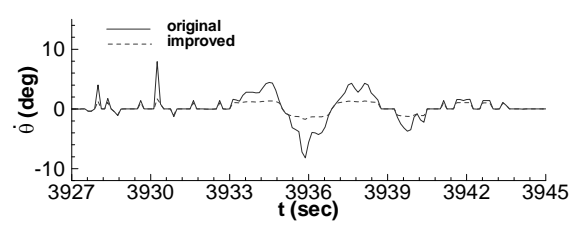

FIGURE X. ORIGINAL AND IMPROVED TIME RATE OF PITCH ANGLE

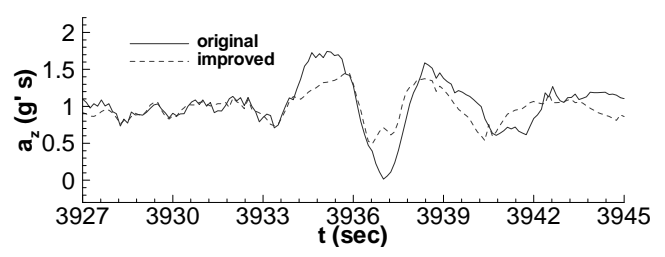

FIGURE XI. ORIGINAL AND IMPROVED VERTICAL ACCELERATIONS

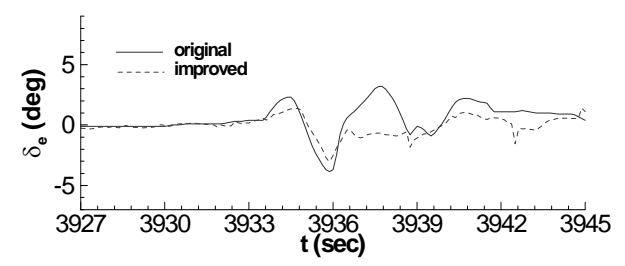

FIGURE XII. ORIGINAL AND IMPROVED ELEVATOR ANGLES

\section{CONCLUDING REMARKS}

The main objective of this paper was to present a practical study of artificial intelligence applications to mitigate the hazards for transport aircraft in severe atmospheric turbulence. A twin-jet transport had large variations in heading angles due to the crosswind effects before the severe atmospheric turbulence encountered. The aileron input ineffective to control the rolling motion, significant increase in angles of attack, large variations of vertical acceleration, and abrupt drop-off flight altitude were the responses to this transport aircraft during the sudden plunging motion in severe atmospheric turbulence. This paper presented the development of flight control models with the function of nonlinear dynamic inversion based on the flight data extracted from FDR and fuzzy-logic modeling technique. The improved control strategy obtained through the simulations from flight control models. The necessary and appropriate applications of rudder, aileron, and elevator controls were demonstrated in the present paper. The simulated results determined that improved control strategy was capable to mitigate the hazards of transport aircraft in severe atmospheric turbulence.

\section{ACKNOWLEDGMENTS}

This research project is sponsored by a grant, MOST 1052221-E-157-006-, from Ministry of Science and Technology (MOST), Taiwan.

\section{REFERENCES}

[1] R. C. Chang, C. E. Ye, C. E. Lan, and Y. L. Lee, Nonlinear and Dynamic Aerodynamic Models for Commercial Transport Aircraft with Adverse Weather Effects, Mathematical Problems in

[2] R. C. Chang, C. E. Ye, C. E. Lan, and W. L. Guan, Stability Characteristics for Transport Aircraft Response to Clear-Air Turbulence, Journal of Aerospace Engineering, Vol. 23, No. 3, July 2010, pp. 197-204.

[3] S. Tan, R. C. Chang, C. E. Lan, and W. L. Guan, Comparative Analysis of Flight Dynamic Characteristics for Transport Aircraft in Severe Atmospheric Turbulence, Journal of Aviation Safety and Management, Vol., 1, No. 2, April 2014, pp. 103-129.

[4] J. Montero, D. Gómez, and H. Bustince, On the Relevance of Some Families of Fuzzy Sets, Fuzzy Sets and Systems, Vol. 158, No. 22, 16, 2007, pp. 2429-2442.

[5] J. Tan, H. Xie, and Y. C. Lee, Efficient establishment of a fuzzy logic model for process modeling and control, IEEE Transactions on Semiconductor Manufacturing, Vol. 8, No. 1, 1995, pp. 50-61.

[6] J. Joh, Y. H. Chen, and R. Langari, On the stability issues of linear Takagi-Sugeno fuzzy models, IEEE Transactions on Fuzzy systems, Vol. 6, Nov. 3, Aug. 1998, pp. 402-410.

[7] Z. Wang, J. Li, C. E. Lan, and J. M. Brandon, Estimation of Unsteady Aerodynamic Models from Flight Test Data, AIAA Paper 2001-4017, American Institute of Aeronautics and Astronautics, Reston, Virginia, USA, 2001.

[8] E. Lan and R. C. Chang, Chapter 7 Fuzzy-Logic Analysis of the FDR Data of a Transport Aircraft in Atmospheric Turbulence, Part 2, Fuzzy Logic-Emerging Technologies and Applications, InTech - 51000, 2012, ISBN 978-953-51-0337-0, Rijeka, Croatia.

[9] H.T. Huyuh, Accurate Monotone Cubic Interpolation, SIAM Journal on Numerical Analysis, Vol. 30, issue 1, 1993, pp. 57-100. 